\title{
马占相思夜间树干液流的分配及其 对整树蒸腾估算的影响
}

\section{王 华 ${ }^{12}$ 赵 平 ${ }^{*}$ 蔡锡安 $^{1}$ 王 权 ${ }^{3}$ 马 玲 饶兴权 $^{1}$ 曾小平 ${ }^{1}$ \\ ( 1 中国科学院华南植物园鹤山丘陵综合试验站, 广州 510650) \\ (2 中国科学院研究生院 北京 100049) (3 静冈大学农学部, 静冈 422-8529 日本)}

\begin{abstract}
摘 要 采用 Granier 热消散探针测定了马占相思 (Acacia mangium) 的树干液流, 结合 Li-6400 光合测定系统测定的 夜间叶片气孔导度和蒸腾 将夜间液流区分为夜间树干水分补充和叶片气孔蒸腾。叶片的蒸腾作用微弱, 因此, 夜 间液流主要用于补充咜水部位的水分亏缺。马占相思夜间水分补充量年内和年际的变化不明显 树形特征的差异 是解释夜间水分补充量变化的重要因子, 夜间水分补充量对于整树蒸腾量的贡献因季节和树木径级的不同而有明 显变化,但对整树总蒸腾量计算造成的误差可以忽略。

关键词 马占相思 Granier 热消散探针法 夜间液流 夜间蒸腾

\section{PARTITIONING OF NIGHT SAP FLOW OF ACACIA MANGIUM AND ITS IMPLICA- TION FOR ESTIMATING WHOLE-TREE TRANSPIRATION}

\author{
WANG Hua $^{1}{ }^{2}$, ZHAO Ping $^{1 *}$, CAI Xi-An ${ }^{1}$, WANG Quan ${ }^{3}$, MA Ling ${ }^{1}$, RAO Xing-Quan ${ }^{1}$, and ZENG Xiao- \\ Ping $^{1}$ \\ ${ }^{1}$ Heshan Hilly Land Interdisciplianry Experimental Station, South China Botanic Garden , Chinese Academy of Sciences , Guangzhou 510650 , China , \\ ${ }^{2}$ Graduate University of Chinese Academy of Sciences , Beijing 100049 , China , and ${ }^{3}$ Institute of Silviculture , Shizuoka University , Shizuoka 422- \\ 8529 , Japan
}

\begin{abstract}
Aims This study was designed to analyze the partitioning of night sap flow in Acacia mangium and its implication for estimating whole-tree transpiration.

Methods Analyses were performed on the partitioning of night sap flow into refilling of internal water storage and transpiration in A. mangium. Sap flow of trees was monitored continuously with Granier' s sensors for estimating whole-tree transpiration. Night sap flow data were used to calculate trunk water recharge. Possible night transpiration and stomatal conductance at leaf level in the canopy were measured with a Li-6400 photosynthesis measuring system.

Important findings Vapor pressure deficit $(V P D)$ and wind speed $(V)$ could not fully explain sap flow in A. mangium at night (night leaf transpiration and stomatal conductance were weak). Therefore, night sap flow of mature $A$. mangium trees was mainly associated with water recharge in the trunk. No significant change in night water recharge of the trunk was found at both seasonal and inter-annual scales. Morphological features of trees including diameter at the breast height $(D B H)$, tree height , and canopy size could explain variances of night water recharge. Furthermore, the error caused by night water recharge on whole-tree transpiration was negligible. The mechanisms and ecological implications of night sap flow partitioning of $A$. mangium deserve further investigation.
\end{abstract}

Key words Acacia mangium, Granier's thermal dissipation probe method, night sap flow , night transpiration

树木的蒸腾耗水是研究森林生态系统功能过程 的一个重要方面。长期以来, 人们采用测定叶片或 枝条蒸腾作用的方法, 通过尺度扩展来研究整树或
者林分的蒸腾耗水,其结果与真实值有较大的偏差， 实际应用也比较困难 (王华田和马履一，2002)。树 干液流测定系统可以直接测定整树蒸腾, 其误差小、 
无破坏性, 并能长期进行连续性的观测 (马玲等， 2005a 赵平等,2005 2006a)。目前较常用的 Granier 树干液流测定系统灵敏度较高, 即使对微弱的液流 活动(如夜间的液流)也能够精确地测定 (赵平等, 2006a)。然而，以往人们对夜间树干液流 ( Night sap flow)给予了较少的关注。夜间液流对于树木的许多 生理过程有重要意义, 譬如, 它与夜间蒸腾有关, 有 助于夜间自根系向上运输物质 (McDonald et al. , 2002), 可对树体内的水分亏缺进行必要的水分补充 (Water recharge), 并可为植物器官的夜间呼吸提供 氧传递的机制 (Daley \& Phillips，2006) 等。Daley 和 Phillips (2006) 在纸桦 (Betula papyrifera)、红橡树 (Quercus rubra) 和红枫 (Acer rubrum) 上观察到的夜间 液流, 大部分用于补充咜水部位的水分亏缺, 夜间蒸 腾可能是纸桦较深边材中为细胞呼吸传递氧的机 制。Lopushinsky (1986)和 Caspari 等(1993)也把夜间 液流归因于对木质部水分亏缺的补充。上述研究虽 注意到夜间液流现象, 但并没有将夜间液流区分为 树干水分补充和夜间蒸腾, 对树木夜间液流分配及 其对总蒸腾量估算的影响认识依然十分有限。

本研究利用 Granier 热消散探针研究造林树种 马占相思 (Acacia mangium) 夜间树干液流的分配, 探 讨其夜间水分补充现象, 分析这种现象的限制因子 及夜间水分补充量的年际变化, 以期为精确估算马 占相思林总蒸腾量、冠层气孔导度和进一步认识冠 层蒸腾与树干液流所代表的蒸腾之间的时滞提供实 验证据。

\section{1 材料和方法}

\section{1 样地概况和实验材料}

野外观测在中国科学院鹤山丘陵综合试验站 $\left(22^{\circ} 40^{\prime} \mathrm{N}, 112^{\circ} 54^{\prime} \mathrm{E}\right)$ 进行。该试验站位于广东省中 部的鹤山市境内, 属于典型的南亚热带丘陵山地, 地 带性土壤为砖红壤。该地区气候温暖, 全年无霜, 年 平均气温为 $21.7{ }^{\circ} \mathrm{C}$, 平均最低气温出现在 1 月, 平 均最高气温出现在 7 月; 雨水充沛, 年降雨量为 $1700 \mathrm{~mm}$, 但降雨分配不均, 有旱、湿季之分, 11月～ 翌年 1 月 (如果没有台风, 有时从 10 月开始)雨水较 少, 但是对植物的生长并不构成威胁, 其它月份皆有 较大降雨。全年太阳辐射强烈, 年平均太阳辐射强 度为 $4.35 \times 10^{5} \mathrm{~J} \mathrm{~cm}^{-2}$, 年平均日照时数为 1797.8 $h($ 马玲等，2005b; 赵平等，1990，2005)。

观测样地位于站内一朝东向的坡地, 海拔约 80 $\mathrm{m}$,马占相思人工纯林的种植株行距为 $3 \mathrm{~m} \times 3 \mathrm{~m}$, 树
龄 20 年,每年 12 月至翌年 2 月有少量落叶。 2003 年 8 月, 选取生境条件比较一致、生长状况良好的 12 株马占相思作为样树, 样树之间的相互干扰比较 小。

\section{2 树干液流密度的测定}

树干液流密度 (Sap flux density)的测定采用法国 学者 Granier 发明的热消散探针法 (Granier, 1987 ; 赵 平等,2006a)。用该方法采集数据, 具有准确、稳定 的优点, 并可连续地读取数据, 因而数据具有系统性 (Granier, 1987)。选取的 12 株马占相思样树 (编号 $1 \sim 12$ ), 胸径 $(D B H)$ 为 $0.13 \sim 0.38 \mathrm{~m}$ (马玲等, $2005 b$; 赵平等, 2006b)。于树干的胸高处 $(1.3 \mathrm{~m}$ 高)，将套入铝管的一对长 $20 \mathrm{~mm}$ 含铜镍合金热电 耦的圆柱形 Granier 热消散探针相隔约 $10 \mathrm{~cm}$ 垂直插 入边材。1 4 号树分别在树干的东、南、西、北 4 个 方位安装探针;5号树在南、北两个方位安装探针; 6 12 号树只在北方位安装探针 (赵平等, 2005)。 样地内的马占相思边材厚度一般为 $1.5 \sim 3.0 \mathrm{~cm}$ 绝 大多数为 $2.0 \mathrm{~cm}$ 左右, 因此我们用探针长度 $(2.0$ $\mathrm{cm})$ 上的液流密度平均值来代表整个边材厚度上的 液流密度平均值 (赵平等, 2006b; 马玲等, 2007)。 为防止机械损伤，我们用塑料盖保护探针，外部包裹 太阳膜, 以减少热辐射和防止雨水浸入。观测系统 的工作原理、安装以及测定程序参见马玲等 (2005a) 和赵平等 $(2005,2006 a)$ 。所用探针由赵平博士依据 Granier 探针原理在美国杜克大学自制，上下探针的 温差电势用数据自动采集仪 (Delta-T data logger, Delta-T , UK) 自动记录(每 $30 \mathrm{~s}$ 测 1 次, 每 $10 \mathrm{~min}$ 进 行平均并储存数据), 据 Granier 建立的以下经验公 式将记录的温差电势转换为液流密度值 :

$$
J_{\mathrm{s}}=119 \times\left(\left(T_{\mathrm{m}}-\Delta T\right) / \Delta T\right)^{1.231}
$$

式中, $J_{\mathrm{s}}$ 为瞬时液流密度 $\left(\mathrm{g} \mathrm{H}_{2} \mathrm{O} \mathrm{m} \mathrm{m}^{-2} \mathrm{~s}^{-1}\right), \Delta T_{\mathrm{m}}$ 为 上下探针之间的最大昼夜温差, $\Delta T$ 是瞬时温差, 此 公式适用于任意树种 (Granier, 1987)。本研究借助 Baseliner 3.0 计算机软件 (由美国杜克大学环境与地 球科学学院 Yavor Parashkevov 博士开发研制)，将从 数据采集仪直接卸载的原始电压数据转换成连续性 的液流密度值 (赵平等, 2006a)。

\section{3 环境因子的测定}

环境因子监测传感器包括监测林内土壤 (距离 地表 $30 \mathrm{~cm}$ 深处) 含水量 $\left(\theta, \mathrm{m}^{3} \cdot \mathrm{m}^{-3}\right)$ ML2x 型土壤 湿度 $(\mathrm{SM})$ 传感器 (3 套)、电热调节式空气温度 $(\mathrm{T})$ 传 感器、Li-6400 光合有效辐射 (PAR) 传感器、HMP35E 空气湿度 $(R H)$ 传感器 (2 套), 除土壤湿度传感器安 
装在马占相思样地并与树干液流数据的采集仪相接 外, 其它环境因子监测传感器均安装在距样地 $50 \mathrm{~m}$ 处的气象站空旷地上, 监测频度与树干液流的测定 同步。风速数据由中国科学院鹤山丘陵综合试验站 $\left(22^{\circ} 40^{\prime} \mathrm{N}, 112^{\circ} 54^{\prime} \mathrm{E}\right)$ 提供。

\section{4 边材面积的确定}

边材面积是计算整树蒸腾的关键参数。为避免 伤害样树而影响液流的测定, 在样地外围选取 24 株 胸径大小有代表性的样株。样株选择标准 选取生 境条件比较一致，生长状况良好的样树，彼此之间的 相互干扰比较小。径级分布: 通过对马占相思林样 地树木径级的分布频度及对应的边材总边材面积的 分析，把马占相思样地树木的径级分为 5 个: $D B H<$ $0.15 \mathrm{~m} 、 0.15 \mathrm{~m}<D B H<0.20 \mathrm{~m} 、 0.20 \mathrm{~m}<D B H<$ $0.25 \mathrm{~m} 、 0.25 \mathrm{~m}<D B H<0.30 \mathrm{~m}$ 和 $0.30 \mathrm{~m}<D B H$ 。 分别量取胸径和树皮厚度, 然后在树干的胸高位置 去除树皮, 用林业用的生长锥打孔器钻取直径为 5 $\mathrm{mm}$ 、深度为胸径的 $1 / 2$ 的木栓, 由于马占相思边材 与心材的颜色易于区分, 目测可直接判断木栓两区 域的分界线, 确定边材的厚度 (赵平等, 2005 ; 马玲 等, 2007)。分析发现，马占相思边材面积 $\left(A_{\mathrm{s}}\right)$ 与胸 径之间存在极显著的幂函数关系, 据此建立边材面 积与胸径的关系式 :

$$
A_{\mathrm{s}}=\mathrm{k}(D B H)^{\mathrm{b}}
$$

式中 , $\mathrm{k}$ 和 $\mathrm{b}$ 是通过非线性回归分析得出的系数, 本 研究的分析结果分别为 $: k=0.1930$ 和 $b=1.1844$, 以此关系式计算 12 株样树的边材面积。

\section{5 夜间水分补充量的计算}

夜间水分补充量的计算公式为：

$W=\Sigma\left(J_{\mathrm{s}} \times A_{\mathrm{s}} \times t\right)$

式中, $W$ 为夜间水分补充量 $(\mathrm{g}) ; J_{\mathrm{s}}$ 是夜间光合有效 辐射为 0 时的液流密度值 $\left(\mathrm{g} \mathrm{H}_{2} \mathrm{O} \mathrm{m} \mathrm{m}^{-2} \mathrm{~s}^{-1}\right) ; A_{\mathrm{s}}$ 为 边材面积 $(\mathrm{m})$ 。根据数据采集频度, 每个 $J_{\mathrm{s}}$ 是 10 $\min$ 液流密度的平均值，因此，时间 $t$ 为 $600 \mathrm{~s}$ 。

1.6 马占相思夜间叶片气孔导度和蒸腾速率的测 定

为了测定马占相思夜间叶片的气孔导度和蒸腾 速率、判断所测定的树干液流与夜间蒸腾的关系, 我 们在样地上选取 3 株位置合适、生长良好的马占相 思，从每株选取 3 枚健康的叶片，采用拉枝法，于 2006 年 7 月 $18 \sim 19$ 日连续两个晴天的夜间, 用 Li6400 光合测定系统原位测定活体叶片的气孔导度 和蒸腾速率 测定频度 : 17:00 19:00 时段每 $30 \mathrm{~min}$ 测定 1 次 ,19:00 时至次日清晨 7:00 每小时测定 1
次。

\section{7 统计分析}

用 SPSS11.5 统计软件进行数据分析，用单因素 方差分析夜间水分补充量的年内变化，树干夜间水 分补充量与胸径、树高和冠幅之间的回归关系采用 曲线参数估计法, 夜间液流密度和树干夜间水分补 充量与相应的主要环境因子之间的相关关系采用相 关分析和回归分析。

\section{2 结果与分析}

\section{1 马占相思树干液流密度的日变化}

图 1 表示 2004 年 7 月被观测样树液流密度均 值、最大和最小径级代表性树木的液流密度日变化。 从图 1 可知, 无论树木径级大小, 夜间均观察到真实 的液流; 与白天树干的液流密度相比, 夜间液流密度 较低, 夜间液流密度值的变异较小。

\section{2 夜间树干液流与冠层蒸腾}

2.2.1干湿季夜间树干液流与水汽压亏缺和风速 之间的关系

环境因子如空气水汽压亏缺和风速都会影响植 物的冠层蒸腾量, 因此, 要想判断马占相思夜间液流 是否主要用于蒸腾, 可以通过分析夜间液流与当时 的空气水汽压亏缺和风速的相关关系来了解, 如果 这种相关关系显著, 并可充分解释夜间液流的变化， 那么所观察到的液流主要是用于夜间蒸腾; 如果这 种关系不显著 或者虽然显著, 但统计学分析结果不 能充分解释夜间液流的变化, 那么液流主要是用于 树干的水分补充 (Daley \& Phillips, 2006 ; Benyon， 1999)。

表 1 指示马占相思夜间液流密度与水汽压亏 缺、风速之间的相关关系:无论是旱季还是湿季, 夜 间液流密度与水汽压亏缺、风速之间均呈显著正相 关关系。这表明液流对于夜间蒸腾有一定贡献。表 2 是马占相思夜间液流密度与水汽压亏缺、风速之 间的曲线参数估计结果，由表 2 可知 无论是旱季还 是湿季, 虽然其液流密度与水汽压亏缺之间拟合的 直线方程是显著的, 但是决定系数 ( $R^{2}$ 值) 很小, 说 明夜间水汽压亏缺未能充分解释液流的变化; 其液 流密度与风速之间拟合的直线方程不具显著性，加 之决定系数 ( $R^{2}$ 值) 很小, 说明夜间风速末能充分解 释液流的变化。总之, 水汽压亏缺和风速等环境因 子可以影响马占相思夜间蒸腾量和蒸腾时间, 却不 能充分解释其夜间液流的变化。

2.2.2 利用热消散法和 Li-6400 测定法研究马占相 

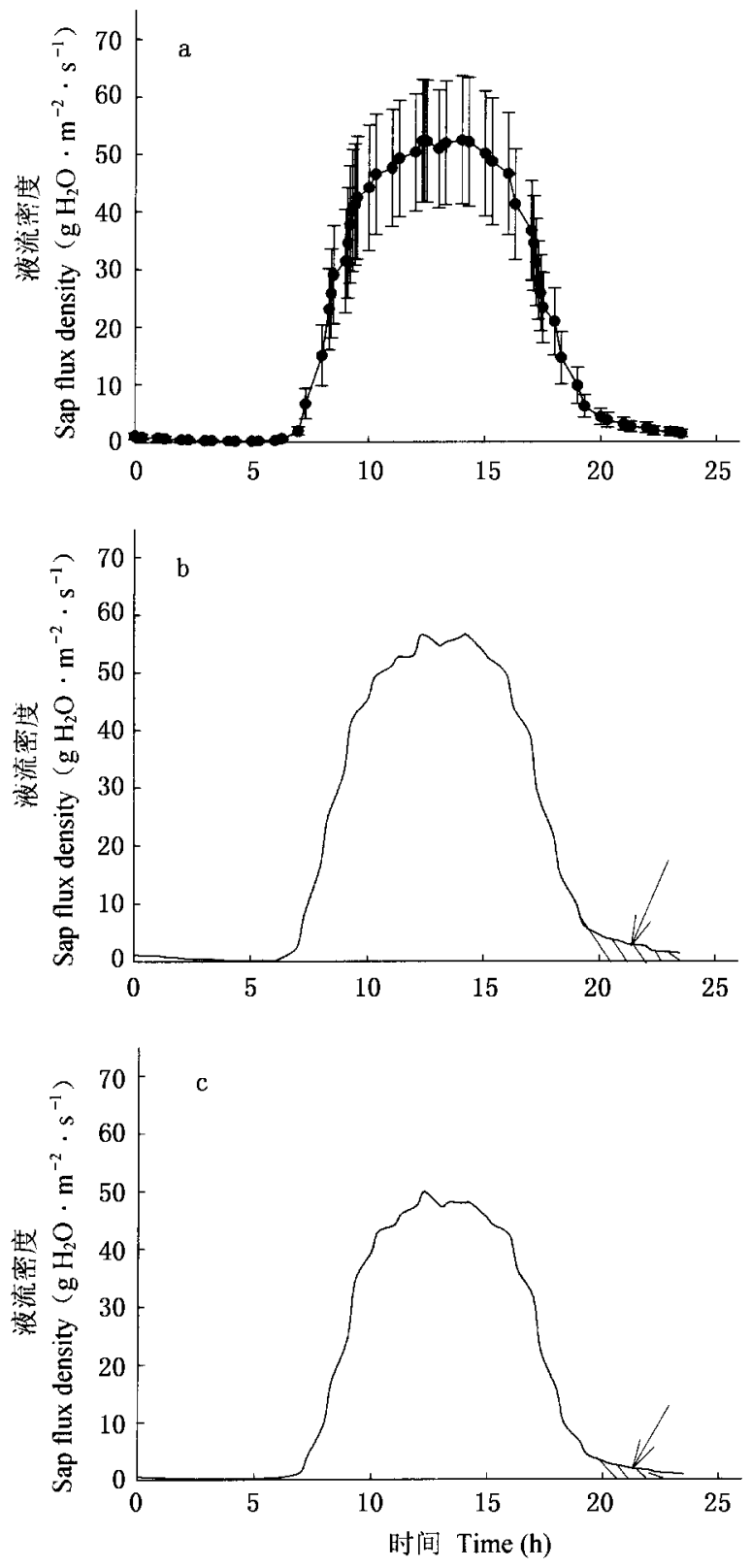

图 1 马占相思平均液流密度的日变化 (a) 和最大径级 (b , DBH = $32.9 \mathrm{~cm})$ 、最小径级 $(\mathrm{c}, D B H=16.9 \mathrm{~cm})$ 代表树木液流密度的日变化 (2004 年 7 月)

Fig.1 Daily variations of average sap flux density of all Acacia mangium sample trees ( $\mathrm{a})$, of the largest tree $(\mathrm{b}, D B H=32.9 \mathrm{~cm})$ and of the smallest tree $(\mathrm{c}, D B H=16.9 \mathrm{~cm})$ in July 2004

$D B H$ : 胸径 Diameter at breast height 箭头指向的阴影区代表夜 间液流 The shadow sections to which arrows pointed represent night sap flow

思夜间水分利用的时间动态

为了进一步阐明马占相思夜间液流的功能, 于 2006 年 7 月 $18 \sim 19$ 日采用 $\mathrm{Li}-6400$ 光合测定系统连 续两天原位测定了活体叶片蒸腾速率的变化, 并取 得同期的液流密度数据, 结果如图 2 和图 3 所示。
从图 2 可知 : 17:00 19:00 光合有效辐射逐渐下降 至零，气孔逐渐关闭，蒸腾速率不断减小; 19:00 至 次日 6:00, 气孔接近关闭, 蒸腾速率也很低, 二者变 化均较平稳。由图 3 可见，利用热消散法测定的液 流密度与采用 Li-6400 光合测定系统测定的蒸腾速 率之间有很好的相关性(检验水平取 $0.05, R^{2}$ 高达 $0.577, p=0.002)$, 说明夜间液流可以较好地解释用 Li-6400 测得的夜间微弱的蒸腾速率。

尽管夜间蒸腾非常微弱, 但是为给下文中树干 咜水的估算提供依据，我们采用折线图直观地表示 了 Li-6400 光合测定系统测定的蒸腾速率以及热消 散法测定的液流密度随时间变化的规律 (图 4)。如 图 4 所示, 在夜间绝大部分时间里, 消散法测定的液 流密度值都远远高于 Li-6400 光合测定系统测定的 蒸腾速率。考虑到夜间蒸腾作用微弱的事实, 我们 有理由推断: 实际所观察到的马占相思夜间液流很 可能主要用于树干水分补充, 这是因为 7 月份华南 丘陵地区处于湿季, 白天蒸腾量很大 极易引起树体 内水分亏缺, 故夜间需要补充水分。

2.3 马占相思夜间水分补充量的年内变化及其影 响因素

\subsection{1 马占相思夜间水分补充量的年内变化}

表 3 为 2004 年和 2005 年马占相思夜间水分补 充量年内变化的单因素方差分析,结果显示,2004 年马占相思夜间水分补充年内各月份之间均没有极 显著差异 $(2004$ 年,$s i g .=0.036>0.01) ; 2005$ 年马 占相思夜间水分补充年内各月份之间均没有显著差 异(2005 年, sig. $=0.275>0.05)$, 这意味着马占相 思夜间水分补充量受环境因子影响的可能性不大。

2.3.2 马占相思夜间水分补充量与环境因子之间 的关系

由表 4 和表 5 可知, 2004 年夜间水分补充量与 土壤湿度和大气湿度之间显著相关, 两者与夜间水 分补充量拟合的直线方程是显著的, 但是决定系数 $R^{2}$ 分别是 0.211 和 0.053 ,也就是说, 虽然土壤和大 气湿度的变化与夜间水分补充量变化相似, 但它们 并不是决定夜间水分补充量的重要因子。2005 年， 马占相思树干夜间水分补充与大气温度之间也呈显 著相关关系, 但大气温度对夜间水分补充量的决定 系数极低 $\left(R^{2}\right.$ 仅为 0.064$)$, 说明在实验样地所处的 气候条件下, 温度也不是决定夜间水分补充量的重 要因子。

2.3.3 马占相思树干夜间水分补充量与树形因子 之间的回归分析 
表 12005 年旱季与湿季马占相思夜间液流密度与水汽压亏缺、风速之间的相关分析

Table 1 Correlations between night sap flux density and vapor pressure deficit, wind speed of Acacia mangium in the dry and wet seasons of 2005

\begin{tabular}{cccc}
\hline $\begin{array}{c}\text { 夜间液流密度 } \\
\text { Night sap flux density }\left(\mathrm{g} \mathrm{H}_{2} \mathrm{O} \mathrm{m}^{-2} \mathrm{~s}^{-1}\right)\end{array}$ & $\begin{array}{c}\text { 水汽压亏缺 } \\
\text { Vapor pressure deficit }(\mathrm{kPa})\end{array}$ & $\begin{array}{c}\text { 风速 } \\
\text { Wind speed }\left(\mathrm{m} \mathrm{s} \mathrm{s}^{-1}\right)\end{array}$ \\
\hline 旱季 Dry season & 泊松相关系数 Pearson correlation & $0.237^{* *}$ & $0.118^{* *}$ \\
& 显著度 Sig. (2-tailed) & 0.000 & 0.000 \\
& 样本数 $n$ & 2015 & 1961 \\
湿季 Wet season & 泊松相关系数 Pearson correlation & $0.236^{* *}$ & $0.101^{* *}$ \\
& 显著度 Sig. (2-tailed) & 0.000 & 0.000 \\
& 样本数 $n$ & 2196 & 1300 \\
\hline
\end{tabular}

** 相关关系在 0.01 水平上显著 (双尾检验) Correlation is significant at the 0.01 level (2-tailed)

表 2 夜间液流密度与水汽压亏缺、风速之间的曲线参数估计

Table 2 Curve parameter estimation between night sap flux density and vapor pressure deficit (VPD), wind speed

\begin{tabular}{|c|c|c|c|c|}
\hline $\begin{array}{c}\text { 因变量 } \\
\text { Dependent }\end{array}$ & $\begin{array}{c}\text { 自变量 } \\
\text { Independent }\end{array}$ & $\begin{array}{c}\text { Model fit 方程 } \\
\text { Model fit equation }\end{array}$ & $R^{2}$ & $p$ \\
\hline 夜间液流密度 & 2 月夜间水汽压亏缺 $V P D$ at night in February $(\mathrm{kPa})$ & $y=0.3769+0.1119 x$ & 0.156 & 0.000 \\
\hline \multirow[t]{3}{*}{ Night sap flux density $\left(\mathrm{g} \mathrm{H}_{2} \mathrm{O} \mathrm{m} \mathrm{m}^{-2} \mathrm{~s}^{-1}\right)$} & 2 月夜间风速 Wind speed at night in February $\left(\mathrm{m} \mathrm{s}^{-1}\right)$ & $y=0.7628-0.0293 x$ & 0.005 & 0.191 \\
\hline & 8 月夜间水汽压亏缺 $V P D$ at night in August $(\mathrm{kPa})$ & $y=3.4119+0.5615 x$ & 0.061 & 0.001 \\
\hline & 8 月夜间风速 Wind speed at night in August $\left(\mathrm{m} \mathrm{s}^{-1}\right)$ & $y=3.9267+0.1567 x$ & 0.001 & 0.699 \\
\hline
\end{tabular}

$R^{2}:$ 决定系数 Coefficient of determination

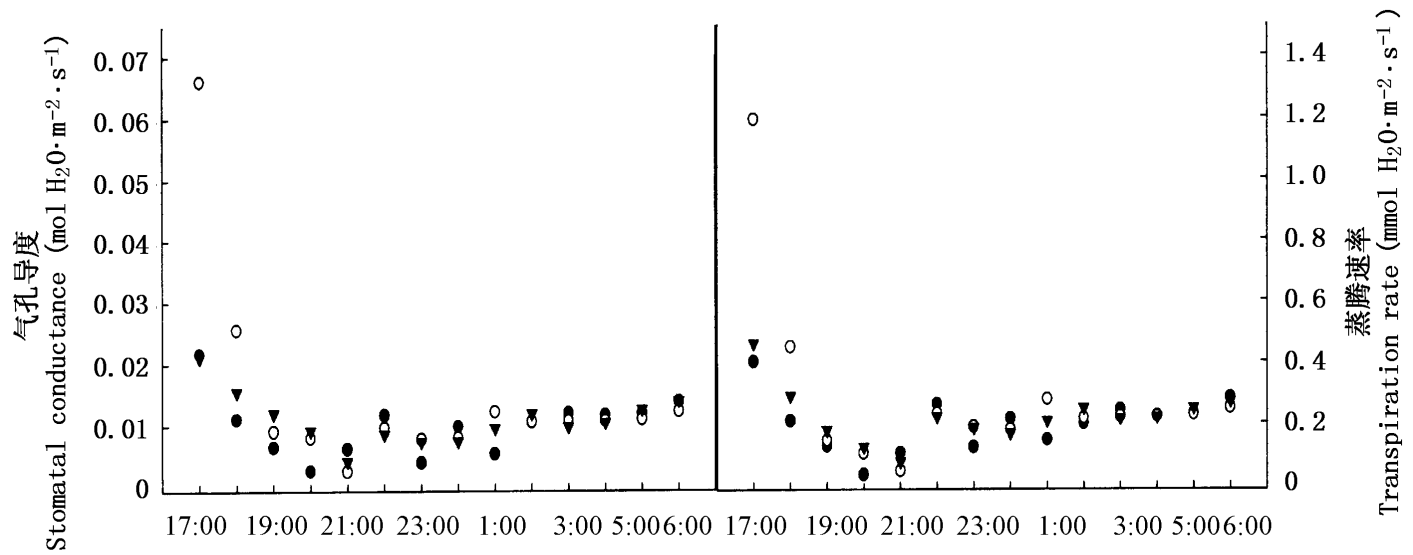

点钟 $0^{\prime}$ clock

图 2 马占相思夜间气孔导度和蒸腾速率的变化

Fig.2 Changes of leaf stomatal conductance and transpiration rate in Acacia mangium during the night

1 号树 Tree No.1 $\bigcirc 2$ 号树 Tree No.2 V 3 号树 Tree No.3

表 3 马占相思树干各月份夜间水分补充量的方差分析

Table 3 One-way ANOVA on monthly night water recharge in Acacia mangium trees

\begin{tabular}{ccccccc}
\hline 年份 & 夜间水分补充量 & 平方和 & 自由度 & 方差 & F \\
Year & Night water recharge $\left(\mathrm{kg} \mathrm{d}^{-1}\right)$ & Sum of squares & $d f$ & Mean square & 显著度 \\
\hline 2004 & 组间 Between groups & 54.171 & 11 & 4.925 & 1.961 & 0.036 \\
& 组内 Within groups & 361.540 & 144 & 2.511 & - & - \\
& 总计 Total & 415.711 & 155 & - & - & - \\
2005 & 组间 Between groups & 40.520 & 11 & 3.684 & 1.228 & 0.275 \\
& 组内 Within groups & 396.054 & 132 & 3.000 & - & - \\
& 总计 Total & 436.574 & 143 & - & - & - \\
\hline
\end{tabular}


由上述分析可知, 环境因子并不能决定马占相 思树干的夜间水分补充量, 夜间水分补充量可能更 多地受到树形因子 (如胸径、树高和冠幅)的影响。 为此, 我们描述夜间水分补充量与胸径、树高和冠幅 之间的回归关系时, 采用曲线参数估计的方法, 通过 调用曲线参数估计法(Curve estimation)模块，生成 11 种模型 (线性模型、对数曲线模型、二次曲线模型、三 次曲线模型、混合曲线模型等), 笁选决定系数 $\left(R^{2}\right)$ 最高的模型拟合 结果如图 5 7 所示。在检验水平 为 0.05 的条件下, 夜间水分补充量和胸径之间的回 归关系的直线拟合最好，决定系数 $R^{2}$ 高达 0.8251 , $p<0.0001(p<0.05)$, 直线方程有显著性意义。夜 间水分补充量和树高之间的回归关系, 指数曲线拟
合最好, 决定系数 $R^{2}=0.2350, p=0.1455(p>$ $0.05)$,指数曲线不具有显著性意义。对于夜间水分 补充量和冠幅之间的回归关系, 直线拟合最好, 决定 系数 $R^{2}=0.6088, p=0.0017(p<0.05)$, 直线有显 著性意义。因此, 胸径、树高以及冠幅可以充分解释 马占相思夜间水分补充年内变化与环境因子变化不 显著的现象。

\section{4 马占相思夜间水分补充量的年际变化}

从表 6 可以看到,2004 年与 2005 年的马占相思 夜间水分补充量没有显著差异 (独立 $t$ 检验中 $s i g .=$ $0.223>0.05)$, 前文的分析结果认为夜间水分补充 的变化主要是受树形因子的影响。在此,我们分析 了2004年和 2005 年样地环境因子的变化,如图 8

表 4 马占相思夜间水分补充量与主要环境因子之间的相关分析

Table 4 Correlation between night water recharge in Acacia mangium and environmental factors

\begin{tabular}{|c|c|c|c|c|c|}
\hline $\begin{array}{l}\text { 年份 } \\
\text { Year }\end{array}$ & $\begin{array}{c}\text { 夜间水分补充量 } \\
\text { Night water recharge } \\
\quad\left(\mathrm{kg} \mathrm{d}^{-1}\right)\end{array}$ & $\begin{array}{c}\text { 土壤湿度 } \\
\text { Soil moisture } \\
\left(\mathrm{m}^{3} \mathrm{~m}^{-3}\right)\end{array}$ & $\begin{array}{c}\text { 大气湿度 } \\
\text { Air humidity } \\
\quad(\%)\end{array}$ & $\begin{array}{c}\text { 大气温度 } \\
\text { Air temperature } \\
\left({ }^{\circ} \mathrm{C}\right)\end{array}$ & $\begin{array}{c}\text { 水汽压亏缺 } \\
\text { Vapor pressure deficit } \\
(\mathrm{kPa})\end{array}$ \\
\hline \multirow[t]{3}{*}{2004} & 泊松相关系数 Pearson correlation & $0.459^{* *}$ & $0.230^{* *}$ & -0.055 & \\
\hline & 显著度 Sig. (2-tailed) & 0.000 & 0.000 & 0.056 & 0.388 \\
\hline & 样本数 $n$ & 252 & 252 & 252 & 252 \\
\hline \multirow[t]{3}{*}{2005} & 泊松相关系数 Pearson correlation & -0.004 & 0.089 & $0.253^{* *}$ & -0.041 \\
\hline & 显著度 Sig.（2-tailed） & 0.956 & 0.221 & 0.000 & 0.594 \\
\hline & 样本数 $n$ & 188 & 191 & 191 & 168 \\
\hline
\end{tabular}

** 相关关系在 0.01 水平上显著 (双尾检验) Correlation is significant at the 0.01 level (2-tailed)

表 5 马占相思夜间水分补充量与主要环境因子之间的曲线参数估计

Table 5 Curve estimation between night water recharge in Acacia mangium and environmental factors

\begin{tabular}{|c|c|c|c|c|c|c|}
\hline $\begin{array}{l}\text { 年份 } \\
\text { Year }\end{array}$ & $\begin{array}{c}\text { 因变量 } \\
\text { Dependent }\end{array}$ & $\begin{array}{c}\text { 自变量 } \\
\text { Independent }\end{array}$ & $R^{2}$ & $\begin{array}{c}\text { 自由度 } \\
d f\end{array}$ & $F$ & $p$ \\
\hline \multirow[t]{4}{*}{2004} & 夜间水分补充量 & 土壤湿度 Soil moisture $\left(\mathrm{m}^{3} \mathrm{~m}^{-3}\right)$ & 0.211 & 250 & 66.85 & 0.000 \\
\hline & Night water recharge $\left(\mathrm{kg} \mathrm{d}^{-1}\right)$ & 大气湿度 Air humidity（\%） & 0.053 & 250 & 13.97 & 0.000 \\
\hline & & 大气温度 Air temperature $\left({ }^{\circ} \mathrm{C}\right)$ & 0.015 & 250 & 3.69 & 0.056 \\
\hline & & 水蒸汽压亏缺 Vapor pressure deficit $(\mathrm{kPa})$ & 0.003 & 250 & 0.75 & 0.338 \\
\hline \multirow[t]{4}{*}{2005} & 夜间水分补充量 & 土壤湿度 Soil moisture $\left(\mathrm{m}^{3} \mathrm{~m}^{-3}\right)$ & 0.000 & 186 & $3.1 \mathrm{E}-03$ & 0.956 \\
\hline & Night water recharge $\left(\mathrm{kg} \mathrm{d}^{-1}\right)$ & 大气湿度 Air humidity (\%) & 0.008 & 189 & 1.51 & 0.221 \\
\hline & & 大气温度 Air temperature $\left({ }^{\circ} \mathrm{C}\right)$ & 0.064 & 189 & 12.89 & 0.000 \\
\hline & & 水蒸汽压亏缺 Vapor pressure deficit $(\mathrm{kPa})$ & 0.002 & 166 & 0.29 & 0.594 \\
\hline
\end{tabular}

$R^{2}:$ 决定系数 Coefficient of determination

表 62004 年和 2005 年间马占相思夜间水分补充量之间的独立 $t$ 检验

Table 6 Independent samples test on night water recharge of Acacia mangium in 2004 and 2005

\begin{tabular}{|c|c|c|c|c|c|}
\hline & \multirow{2}{*}{$\begin{array}{c}\text { 方差齐性 } \\
\text { Equal variances }\end{array}$} & \multicolumn{2}{|c|}{$\begin{array}{c}\text { 方差同质性检验 } \\
\text { Levene' s test for equality of variances }\end{array}$} & \multicolumn{2}{|c|}{$\begin{array}{c}\text { 平均数的 } t \text { 检验 } \\
t \text {-test for equality of means }\end{array}$} \\
\hline & & $F$ & 显著度 Sig. & $t$ & 显著度 Sig. \\
\hline \multirow{2}{*}{$\begin{array}{l}\text { 夜间水分补充量 } \\
\text { Night water recharge }\left(\mathrm{kg} \mathrm{d}^{-1}\right)\end{array}$} & $\begin{array}{l}\text { 假设方差齐性 } \\
\text { Equal variances assumed }\end{array}$ & 0.112 & 0.741 & 1.255 & - \\
\hline & $\begin{array}{l}\text { 假设方差不齐 } \\
\text { Equal variances not assumed }\end{array}$ & - & - & 1.255 & 0.233 \\
\hline
\end{tabular}




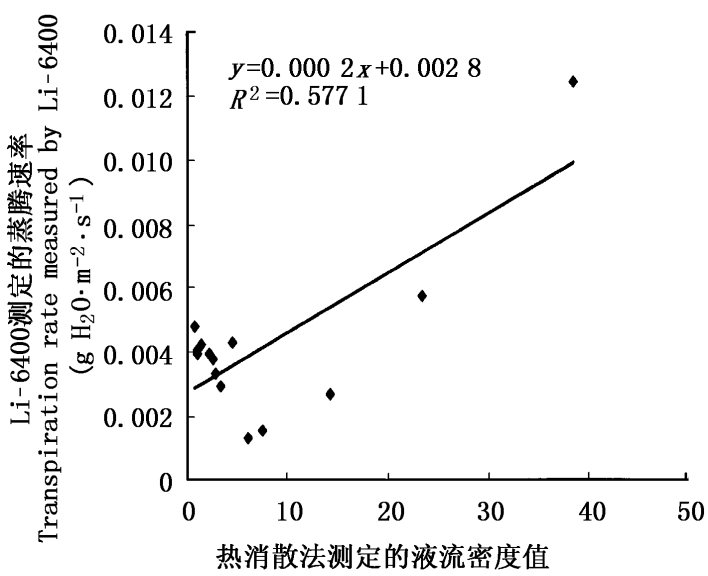

Sap flow density measured by TDP $\left(\mathrm{g} \mathrm{H}_{2} 0 \cdot \mathrm{m}^{-2} \cdot \mathrm{s}^{-1}\right)$

图 3 热消散法测定的液流密度与气体交换法测定的蒸腾速率 之间的回归关系

Fig.3 Regression relationship between the night sap flow density measured

by using the thermal dissipation probe (TDP) method and night

transpiration rate measured by gas exchange method ( $\mathrm{Li}-6400$ )

气体交换法 ( $\mathrm{Li}-6400)$ 测定的蒸腾速率的单位是 $\mathrm{mmol} \mathrm{H}_{2} \mathrm{O} \mathrm{m} \mathrm{m}^{-2}$ $\mathrm{s}^{-1}$ 为了方便比较, 换算为热消散法测定的液流密度值的单位 $\mathrm{g}$ $\mathrm{H}_{2} \mathrm{O} \mathrm{m} \mathrm{m}^{-2} \mathrm{~s}^{-1}$ For the comparison, the unit of transpiration rate $\left(\mathrm{mmol} \mathrm{H}_{2} \mathrm{O}\right.$ $\mathrm{m}^{-2} \mathrm{~s}^{-1}$ ) measured by gas exchange method ( $\left.\mathrm{Li}-6400\right)$ is converted into the unit of sap flow density measured by TDP $\left(\mathrm{g} \mathrm{H}_{2} \mathrm{O} \mathrm{m} \mathrm{m}^{-2} \mathrm{~s}^{-1}\right)$

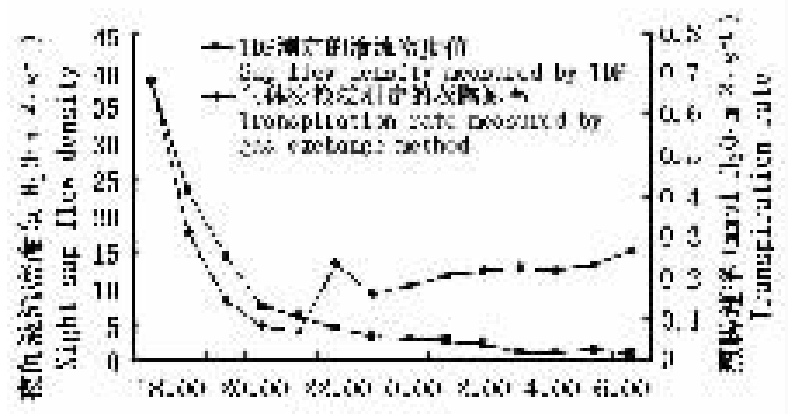

'it 6 elok

图 4 热消散法测定的夜间液流密度与气体交换法测定的夜间 蒸腾速率的时间动态

Fig.4 Temporal dynamics of night sap flow density measured by the thermal dissipation probe method and night transpiration rate measured by gas exchange method

所示, 两年的大气温度、降雨量、大气湿度、水汽压亏 缺的大小和变化规律都比较相似, 因此环境因子也 不会造成马占相思夜间水分补充量年际间的差异。

\section{5 马占相思夜间液流对估测整树蒸腾的影响}

2.5.1 不同径级马占相思夜间水分补充量占日总 液流量比例的季节变化

由图 9 可见，旱季夜间水分补充量占日总液流 量的比例高于湿季, 这是由于两季节夜间水分补充 量之间的差异不显著,而旱季的总蒸腾量小于湿季 的缘故。与径级较大的个体相比，径级较小个体的

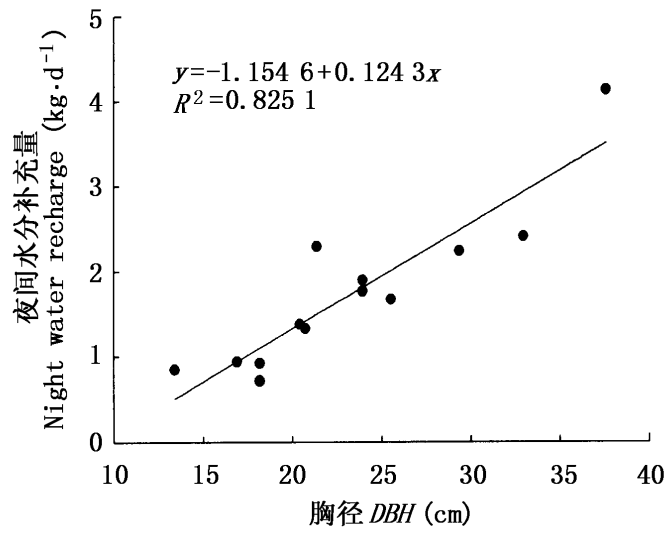

图 5 夜间水分补充量与胸径的回归关系

Fig.5 Regression relationship between night water recharge amount and diameter at breast height $(D B H)$

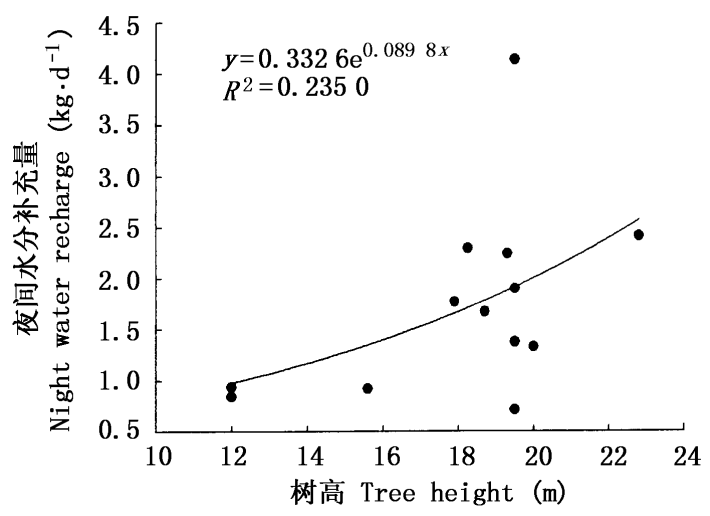

图 6 夜间水分补充量与树高之间的回归

Fig.6 Regression relationship between night water recharge and tree height

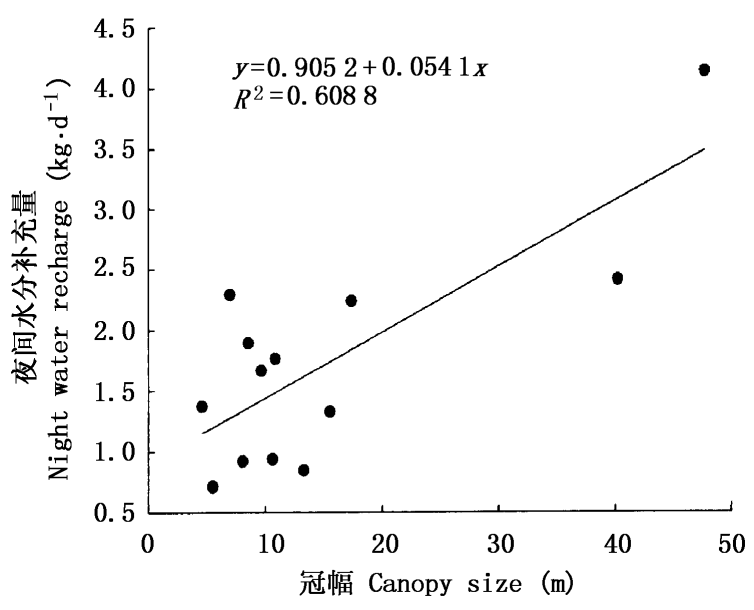

图 7 夜间水分补充量与冠幅之间的回归

Fig.7 Regression relationship between night water recharge and canopy size 


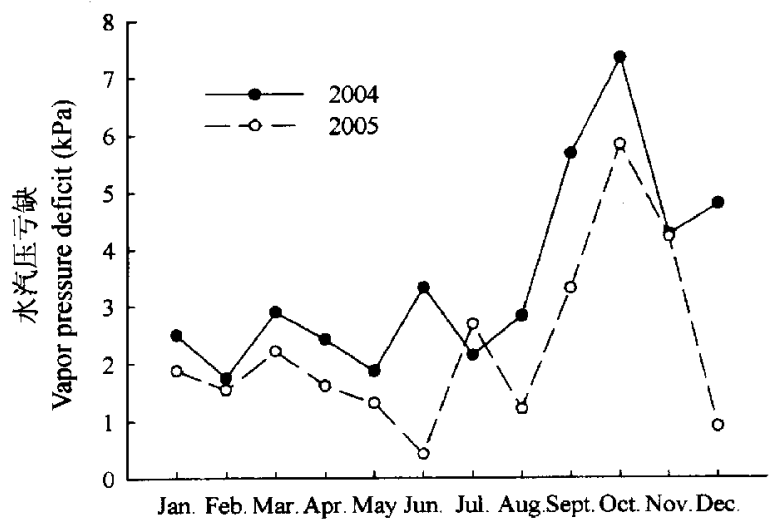

月份 Month

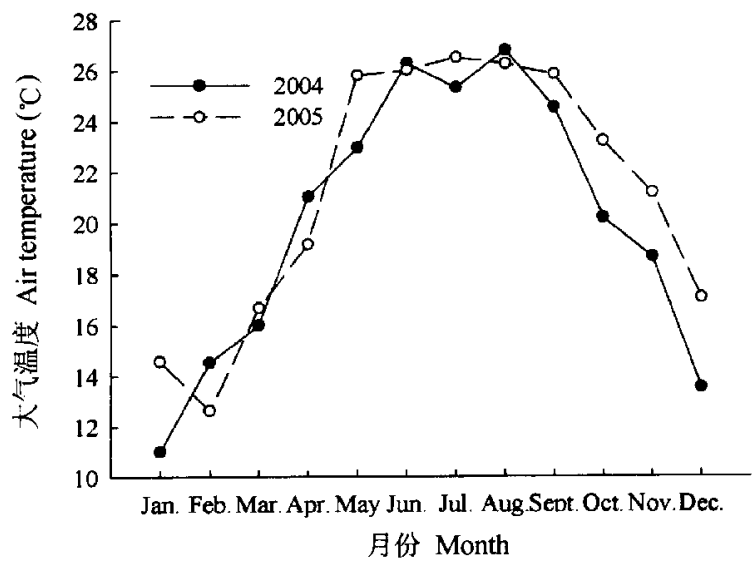

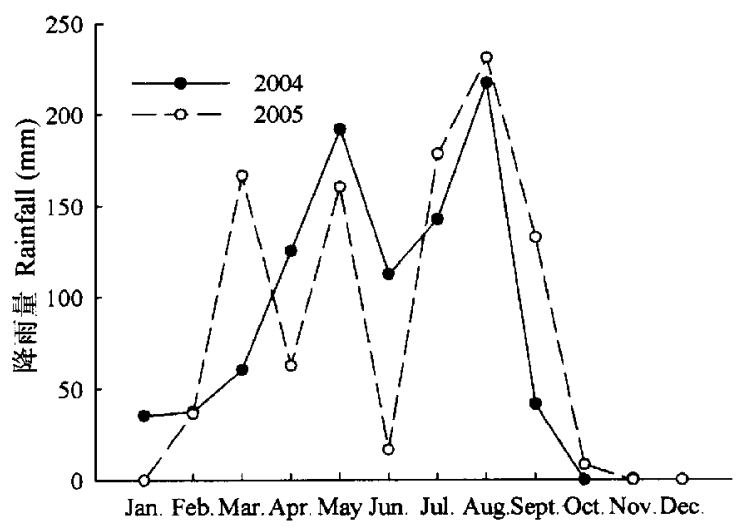

月份 Month

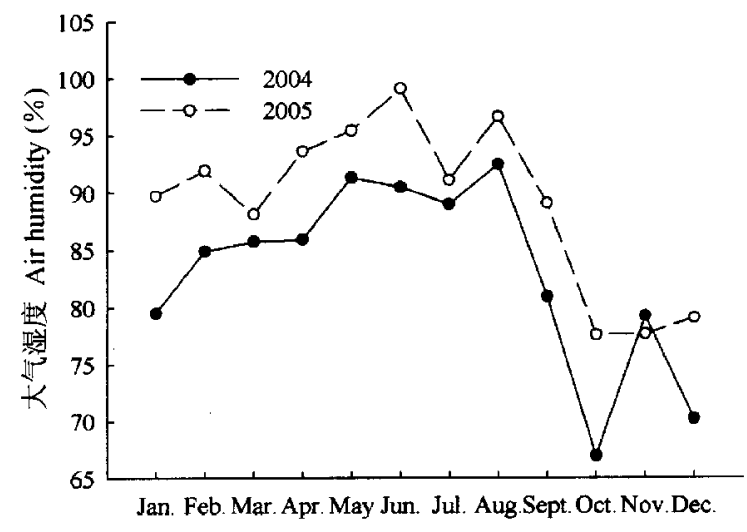

月份 Month

图 82004 年和 2005 年样地主要环境因子变化的比较

Fig. 8 Comparison of major environmental factor changes in sample plot in 2004 and 2005

表 7 马占相思总液流量与除去夜间液流的液流量之间的独立 $t$ 检验

Table 7 Independent samples test between the total sap flow and the sap flow eliminating night sap flow of Acacia mangium

\begin{tabular}{|c|c|c|c|c|}
\hline \multirow{2}{*}{$\begin{array}{c}\text { 方差齐性 } \\
\text { Equal variances }\end{array}$} & \multicolumn{2}{|c|}{$\begin{array}{c}\text { 方差同质性检验 } \\
\text { Levene' s test for equality of variances }\end{array}$} & \multicolumn{2}{|c|}{$\begin{array}{c}\text { 均值的 } t \text { 检验 } \\
t \text {-test for equality of means }\end{array}$} \\
\hline & $F$ & 显著度 $\mathrm{Sig}$. & $t$ & 显著度 Sig. \\
\hline 假设方差齐性 Equal variances assumed & 0.000 & 0.997 & 0.020 & 0.984 \\
\hline 假设方差不齐 Equal variances not assumed & & & 0.020 & 0.984 \\
\hline
\end{tabular}

夜间水分补充量占总蒸腾量的比率略大, 意味着夜 间水分补充量更多的是由树木的树形特征所决定， 除了上面提及的树高、胸径、冠幅等特征外，其生理 活动与营养状况也可能对此产生影响。

2.5.2 马占相思夜间水分补充量对于整树总蒸腾 量估算的误差分析

人们通常利用热消散探针所获得的液流量来代 替冠层蒸腾，进而估算整树乃至林分尺度上的蒸腾 量 (赵平等, 2006a)。与白天的蒸腾相比, 虽然夜间 液流量很小, 但是并不能确定它是否会引起总蒸腾
量估算的误差。图 10 是 2005 年的总蒸腾和日间蒸 腾量 (总蒸腾量减去夜间液流量) 的年内变化, 表 7 是对两者进行独立 $t$ 检验的结果 $(\mathrm{Sig} .=0.984>$ $0.05)$ 结果表明, 如果将夜间液流的测定值计入总 蒸腾,估算整树蒸腾并未产生显著误差。

\section{3 讨 论}

\section{1 马占相思夜间液流的分配}

以往许多利用热消散探针测定树干液流的研 究 极少把夜间液流区分为夜间蒸腾和树木体内的 


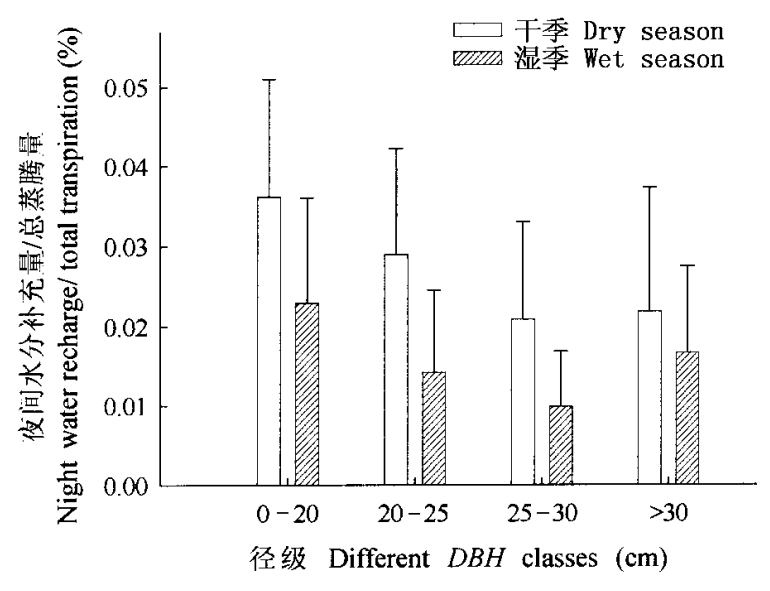

图 92005 年不同径级马占相思夜间水分补充量 与总蒸腾量比率的季节变化

Fig.9 Seasonal changes in the ratio of night water recharge to total transpiration of Acacia mangium at different breast heights in 2005

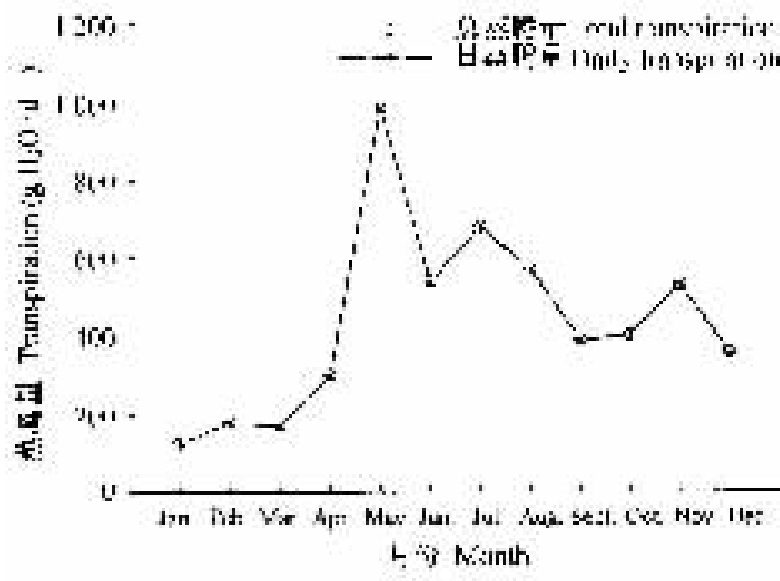

图 102005 年马占相思总蒸腾量和日蒸腾量的年内变化

Fig. 10 Inter-annual changes of total transpiration and daily transpiration of Acacia mangium in 2005

水分补充 或者只是假定夜间叶片的气孔关闭 蒸腾 不明显。但是, 已有越来越多的证据显示，一些植物 的气孔在夜间仍能维持部分开放的状态，如果有足 够的环境因子驱动，夜间蒸腾活动是比较明显的 (Daley \& Phillips，2006)。在美国的北部，生活史、光 合途径、生活习性不同的 17 种植物中，有 11 种植物 夜间的气孔导度和蒸腾作用均能被观测到 (Snyder et al . ,2003) ,我们研究发现，马占相思夜间有微弱 的蒸腾作用和适度的气孔导度, 并维持在一个比较 稳定的水平。环境因子如空气水汽压亏缺和风速会 影响夜间蒸腾量的大小,可以解释桉树 (Eucalyptus grandis)、Malus sylvestris $\times$ Red delicious、猕猴桃 $(A c$ tinidia deliciosa $)$ 和纸桦夜间液流的变化动态 ,因为这
些树种的夜间液流更多地用于夜间蒸腾（Benyon， 1999 ; Green et al . , 1989 ;Daley \& Phillips ,2006)。与 此不同的是, Daley 和 Phillips (2006)发现水汽压亏缺 的变化不能充分地解释红橡树和红枫夜间液流的变 化，这些树种夜间液流更多地分配到树体内水分亏 缺较多的部位, 进行水分补充。本研究结果显示, 水 汽压亏缺和风速均不能充分地解释马占相思夜间液 流的变化, 说明马占相思夜间液流主要用于体内水 分亏缺的水分补充。

虽然本试验同时观察到夜间叶片蒸腾和树干水 分补充的现象，但是前者是不是有一部分来自后者， 对此我们并不清楚, 这需要结合枝条液流的测定, 并 进行科学分析才能获得较清晰的认识。

\section{2 马占相思夜间水分补充现象的分析}

树干液流与树形因子之间的关系已有不少研究 结果 (赵平等, 2006a), 有关学者对 Anacardium excelsum、Cordia alliodora、Ficus insipida 和 Schefflera morototoni 4 种树木水分利用的研究结果显示, 整树蒸腾 耗水随着树木直径的增大显著增加，如胸径 $0.34 \mathrm{~m}$ 的 $C$. alliodora 个体每天蒸腾 $46 \mathrm{~kg}$ 水, 胸径 $0.98 \mathrm{~m}$ 的 A. excelsum 个体每日蒸腾 $750 \mathrm{~kg}$ 水 (Meinzer et $a l$. , 2003) ; 有学者研究 3 种常绿树和两种落叶树 ( Anacardium excelsum、Cecropia longipes、Ficus insipida、 Spondias mombin 和 Luehea seemanni) 的液流发现, 树 干基部日总液流与边材面积和树高显著正相关 (Goldstein et al. , 1998)。但是，针对夜间液流进行 相关分析的研究鲜有报道。马占相思夜间水分补充 量的年内各月份之间和年际间的变化都不显著, 通 过分析它们与环境因子之间的关系, 发现某些环境 因子可以影响马占相思的夜间水分补充量, 但是对 于解释夜间水分补充量的变化并不充分，我们推测 树形因子可以较合理地解释夜间水分补充的现象， 研究结果也显示马占相思的树形特征是解释夜间水 分补充量变化的主要因素。

\section{3 马占相思夜间水分补充的意义}

树木茎干中悾存的水分对调控冠层与大气的水 分交换通量有着重要作用，它对树木蒸腾总量的贡 献变化较大, 从 10\% 20\% (Lostau et al. , 1996; Tanaka \& Kobayashi , 2001, Phillips et al.,2003) 到 30\% 50\% (Holbrook, 2004 ; Waring et al . 1979)。 但是, 低纬度地区如热带树种的相关研究结果比较 少 (Goldstein et al. , 1998)。茎干咜存的水分对日蒸 腾的贡献与环境因子相关, 例如,Pinus pinaster 树干 中咜存的水分在土壤水分充足时占日蒸腾量的 
12\% ,而在干旱的夏末可增加到 $25 \%$ (Lostau et al. , 1996)。马占相思树干夜间水分补充占日总蒸腾的 比例, 旱季较湿季大, 且受环境因子的制约, 与其对 整树蒸腾总量的贡献率是一致的, 说明这个比例在 一定程度上可以反映夜间水分补充对于日蒸腾的贡 献。目前使用的树干液流测定系统通常假定夜间水 分散失为零或者将夜间测定的液流计入蒸腾总量。 对于马占相思而言, 夜间液流对估算整树蒸腾量时 产生的误差是可以忽略的, 但是, 在尺度推移至林分 水平时, 这个误差可能会变得很大。

\section{4 小 结}

本研究利用 Granier 的热消散探针 辅以 Li-6400 光合测定系统对冠层叶片的测定结果, 初步证明马 占相思的树干夜间液流主要用于体内水分亏缺的水 分补充, 并没有对整树蒸腾量的估算造成显著误差。 树形因子可以充分解释马占相思夜间水分补充现象 的年内变化。由于缺乏树枝末端液流的测定, 这种 夜间液流分配机理的研究仍然存在局限性。马占相 思作为植被恢复的先锋树种, 其夜间树干液流的分 配机制和生态学意义还有待于进一步研究。

\section{参 考 文 献}

Benyon RG (1999). Nighttime water use in an irrigated Eucalyptus grandis plantation. Tree Physiology, 19, 853 - 859 .

Caspari HW, Green SR, Edwards WRN (1993). Transpiration of well-watered and water-stressed Asian pear trees as determined by lysimetry, heat-pulse, and estimated by a Penman-Monteith model. Agricultural and Forest Meteorology, 67, 13-27.

Daley M, Phillips NG (2006). Interspecific variation in nighttime transpiration and stomatal conductance in a mixed new England deciduous forest. Tree Physiology, 26, $411-419$.

Goldstein G, Andrade JL, Meinzer FC, Holbrook MN, Cavelier JC, Jackson P, Celis A (1998). Stem water storage and diurnal patterns of water use in tropical forest canopy trees. Plant, Cell \& Environment, 21, 397 - 406.

Granier A (1987). Evaluation of transpiration in a Douglas-fir stand by means of sap flow measurements. Tree Physiology, 3, $309-$ 320 .

Green SR, McNaughton KG, Clothier BE (1989). Observations of nighttime water use in kiwifruit vines and apple trees. Agricultural and Forest Meteorology, 48, $251-261$.

Holbrook NM (2004). Stem water storage. In: Gartner BL ed. Plant Stems: Physiology and Functional Morphology. Academic Press, San Diego, CA, USA, $151-174$.

Lopushinsky W (1986). Seasonal and diurnal trends of heat pulse velocity in Douglas-fir and ponderosa pine. Canadian Journal of Forest Research, 16, 814-821.

Lostau D, Berbiger P, Roumagnac P, Arruda-Pacheco C, David JS, Ferreira MI, Pereira JS, Tavares R (1996). Transpiration of a 64-year-old maritime pine stand in Portugal. I. Seasonal course of water flux through maritime pine. Oecologia, 107, 33-42.
Ma L (马玲), Zhao P (赵平), Rao XQ (饶兴权), Cai XA (蔡 锡安), Zeng XP (曾小平) (2005a). Main determination methods of tree transpiration. Chinese Journal of Ecology (生态学杂 志), 24(1), 88-96. (in Chinese with English abstract)

Ma L (马玲), Zhao P (赵平), Rao XQ (饶兴权), Cai XA (蔡 锡安), Zeng XP (曾小平), Lu P (陆平) (2005b). Effects of environmental factors on sap flow in Acacia mangium. Acta Ecologica Sinica (生态学报), 25, 2145 - 2151. (in Chinese with English abstract)

Ma L (马玲), Rao XQ (饶兴权), Zhao P (赵平), Cai XA (蔡 锡安), Zeng XP (曾小平), Lu P (陆平) (2007). Diurnal and seasonal changes in whole-tree transpiration of Acacia mangium. Journal of Beijing Forestry University (北京林业大 学学报), 29,67-73. (in Chinese with English abstract)

McDonald EP, Erickson JE, Kruger EL (2002). Can decreased transpiration limit plant nitrogen acquisition in elevated $\mathrm{CO}_{2}$ ? Functional Plant Biology, 29, 1115 - 1120 .

Meinzer FC, James SA, Goldstein G, Woodruff D (2003). Wholetree water transport scales with sapwood capacitance in tropical forest canopy trees. Plant, Cell and Environment, 26, 1147 1155 .

Phillips NG, Ryan MG, Bond BJ, McDowell NG, Hinckley TM, Cermak J (2003). Reliance on stored water increases with tree size in three species in the Pacific Northwest. Tree Physiology, $23,237-245$.

Snyder KA, Richards JH, Donovan LA (2003) . Nighttime conductance in $\mathrm{C}_{3}$ and $\mathrm{C}_{4}$ species: do plants lose water at night? Journal of Experimental Botany, 54, $861-865$.

Tanaka T, Kobayashi Y (2001). Water flow and hydraulic characteristics of Japanese red pine and oak trees. Hydrological Processes, 15, $1731-1750$.

Wang HT (王华田), Ma LY (马履一) (2002). Measurement of whole tree's water consumption with thermal dissipation sap flow probe (TDP). Acta Phytoecologica Sinica (植物生态学报), 26, 661 - 667. (in Chinese with English abstract)

Waring RH, Whitehead D, Jarvis PG (1979). The contribution of stored water to transpiration in Scots pine. Plant, Cell and Environment, 2, $309-317$.

Zhao P (赵平), Yu ZY (余作岳), Zeng XP (曾小平) (1990). Water loss by transpiration through leaves of three species of Acacia mangium on hilly land in Heshan, Guangdong Province. Tropical and Subtropical Forest Ecosystem (热带亚热带森林生 态系统) , 7, 12-16. (in Chinese with English abstract)

Zhao P (赵平), Rao XQ (饶兴权), Ma L (马玲), Cai XA (蔡 锡安), Zeng XP (曾小平) (2005). Application of Granier's sap flow system in water use of Acacia mangium forest. Journal of Tropical and Subtropical Botany (热带亚热带植物学报), 13, 457 - 468. (in Chinese with English abstract)

Zhao P (赵平), Rao XQ (饶兴权), Ma L (马玲), Cai XA (蔡 锡安), Zeng XP (曾小平) (2006a). Sap flow-scaled stand transpiration and canopy stomatal conductance in an Acacia mangium forest. Journal of Plant Ecology (Chinese Version) (formerly Acta Phytoecologica Sinica) (植物生态学报)，30, 655 - 665 (in Chinese with English abstract)

Zhao P (赵平), Rao XQ (饶兴权), Ma L (马玲), Cai XA (蔡 锡安), Zeng XP (曾小平) (2006b). The variations of sap flux density and whole-tree transpiration across individuals of Acacia mangium. Acta Ecologica Sinica (生态学报), 26, 4050 4058. (in Chinese with English abstract) 